\title{
Marketing \& Sales oltre la crisi: i cambiamenti in atto nelle medie imprese
}

\author{
Elena Cedrola $^{1}$, Giancarlo Ferrero ${ }^{2}$, Gianluca Gregori $^{3}$
}

\section{Riassunto}

La crisi economica internazionale ha modificato struttura e tendenze di molti mercati. Pochi sono gli studi finora condotti sulle conseguenze per le funzioni di marketing e di vendita. Questo articolo presenta i risultati di un'indagine svolta presso un campione di medie imprese, rivolta a comprendere i cambiamenti in atto nelle strategie di mercato e nel ruolo e nell'importanza attribuita a queste funzioni. I risultati evidenziano il progressivo affermarsi in queste imprese di un più deciso orientamento al mercato, a cui si associa una maggiore valorizzazione di queste funzioni. La funzione di vendita viene a svolgere un ruolo importante anche per l'analisi dei clienti e dei mercati, in una quadro di crescente collaborazione con la funzione di marketing. Permane, tuttavia un significativo gap tra le finalità che si vorrebbe perseguire e le risorse destinate a queste attività.

Parole chiave: Marketing, vendite, medie imprese, strategie competitive, crisi economica, innovazione organizzativa

\begin{abstract}
Marketing \& Sales beyond the crisis: changes occurring in medium-sized companies The international economic crisis has modified structure and trends of many markets. Few studies have been conducted, nowadays, on the consequences for the Marketing and Sales functions. This article presents the results of a survey on a sample of medium-sized companies, aimed at understanding the current changes in market strategies and in the role and importance ascribed to such functions. The results highlight in these companies the emergence of an increased market orientation, associated to a greater valorization of the above mentioned functions. Sales are becoming also important for the analysis of customers and markets, in a context of increasing cooperation with Marketing. Nevertheless, a significant gap remains to be filled between the results to be achieved and the resources to be budgeted for these activities. Keywords: Marketing Sales, Medium size enterprise, Competitive strategies, Economic crisis, Organizational innovation
\end{abstract}

\section{Motivazioni e finalità della ricerca}

Stiamo vivendo un periodo di profonde trasformazioni nell'economia internazionale determinate da molteplici fattori: la crescita impetuosa dell'economia di alcuni paesi (Cina, India, Brasile), che ha accentuato la pressione competitiva,

\footnotetext{
${ }^{1}$ Professore associato di Economia e Gestione delle Imprese, Università di Macerata, elena.cedrola@unimc.it

${ }^{2}$ Professore ordinario di Marketing, Università di Urbino "Carlo Bo", giancarlo.ferrero@uniurb.it

${ }^{3}$ Professore ordinario di Marketing, Università Politecnica delle Marche, g.gregori@univpm.it
} 
ridisegnato per molti aspetti la divisione internazionale del lavoro e nel contempo aperto nuovi sbocchi di mercato; lo sviluppo delle ICT, che hanno creato nuovi canali di comunicazione e di vendita e nuove possibilità di interazione e collaborazione, abbattendo tradizionali barriere connesse alla distanza geografica; la globalizzazione, che ha favorito processi di concentrazione dell'offerta in vari settori e nel contempo ridefinito le modalità di segmentazione dei mercati e l'attrattività dei diversi segmenti; la crescente complessità dei mercati dei beni di largo consumo, caratterizzati da una domanda sempre più frammentata, variabile, consapevole, rivolta verso la ricerca della soddisfazione di nuove esigenze immateriali, condizionata da più stringenti vincoli connessi alla capacità di spesa.

La crisi economica che ha investito nel biennio 2008-2009 tutta l'economia mondiale ha impresso una forte accelerazione a questi processi di cambiamento, determinando per le imprese scenari sempre più incerti, dinamici, difficili da interpretare ma anche caratterizzati da nuove importanti opportunità che possono essere sfruttate.

Le funzioni di marketing e di vendita possono svolgere un ruolo chiave per aiutare le imprese ad orientarsi in questo contesto e per cogliere le nuove possibilità di sviluppo che si prospettano, ma non sempre questa considerazione è condivisa dalle aziende che, anzi, in molti casi hanno deciso di ridurre i budget destinati a queste funzioni durante gli anni della crisi.

Il problema assume una valenza particolarmente critica in Italia, dove il sistema produttivo è caratterizzato da una prevalente presenza di piccole e medie imprese, che in generale non formulano le strategie di mercato mediante approcci sistematici e strutturati, né dispongono di professionalità qualificate in campo commerciale. Come vari studiosi hanno rilevato, il marketing attuato dalle PMI è molto spesso caratterizzato da una interpretazione riduttiva ed incompleta del marketing concept ${ }^{4}$ (Marchini, 2000; Mc Cartan-Quinn e Carson, 2003), che porta ad adottare un approccio di marketing reattivo (e non proattivo), tattico (e non strategico), empirico ed intuitivo (Mattiacci e Ceccotti, 2005). Ciò ha indotto a parlare di un "marketing all'italiana" (Varaldo e al., 2006), originale e non strutturato secondo i canoni della teoria manageriale, che accanto a connotazioni positive presenta notevoli limiti, che possono indebolire nel medio termine la capacità competitiva delle imprese. Indagini svolte (Ferrero e Fortezza, 2007) hanno, però, evidenziato che, pur se prevale un orientamento gestionale al prodotto o al processo, però esistono anche procedure, competenze e canali informativi che aiutano queste imprese ad interfacciarsi con il mercato in modo più consapevole ed efficace di quanto si sarebbe portati a pensare. Dedicano grande attenzione alla cura dei rapporti con i clienti (talvolta a scapito della redditività), mentre non sviluppano adeguate capacità di analisi dell'ambiente competitivo.

Partendo da queste considerazioni ci è sembrato di particolare interesse realizzare, in funzione del VII convegno annuale della Società Italiana di Marketing, un'indagine per comprendere se e come le imprese stanno modificando le strategie di mercato e

\footnotetext{
${ }^{4}$ Ciò perché il marketing attuato dalle PMI è nella maggior parte dei casi frutto del "sapere
} tacito” dell'imprenditore (Marchini, 2000). 
ridefinendo i compiti e l'organizzazione delle funzioni commerciali (marketing e vendite) a seguito dei mutamenti di scenario intervenuti con la crisi economica internazionale.

La ricerca è stata condotta da un gruppo di lavoro comprendente docenti delle facoltà di Economia delle Università di Ancona (Politecnica delle Marche), di Macerata e di Urbino ("Carlo Bo") $)^{5}$ ed è stata presentata al convegno annuale della Società Italiana di Marketing svoltosi ad Ancona il 23-24 settembre 2010.

Nonostante la crisi sia stata oggetto di numerosi studi e pubblicazioni, poche sono le analisi scientifiche finora effettuate su questo tema di ricerca. Un esame da noi condotto sugli articoli pubblicati in 8 riviste scientifiche straniere di marketing ed in 3 riviste italiane ${ }^{6}$ nel triennio 2008-2010 ci ha portato a identificare solo 8 articoli che trattano, con riferimento in genere ad aspetti molto settoriali, l'argomento.

La nostra indagine è stata rivolta ad approfondire quattro aspetti:

1. Le modifiche introdotte nelle strategie di mercato a seguito della crisi economica.

2. I cambiamenti intervenuti nelle politiche di marketing.

3. Le tendenze evolutive nella strutturazione e nel ruolo delle funzioni di marketing e di vendita.

4. I rapporti tra la funzione di marketing e la funzione di vendita.

La finalità perseguita è stata di comprendere la situazione esistente, i processi evolutivi in atto, gli eventuali gap tra le modalità con le quali le imprese sviluppano i processi di marketing e le impostazioni che sarebbe necessario adottare per una efficace gestione dei rapporti con i mercati di sbocco.

Lo studio ha riguardato sia la funzione di marketing che la funzione di vendita, nella convinzione che le due funzioni debbano operare in modo integrato ed interagente (Guenzi e Troilo, 2007), soprattutto nelle $\mathrm{PMI}^{7}$. Non può sfuggire in proposito che il processo dinamico di interazione tra le due funzioni consente di creare maggior valore rispetto a quello che si potrebbe realizzare se queste si comportassero "autonomamente" (Carpenter, 1992; Dawes-Massey, 2005; HamburgJensen, 2007), o ancora peggio - come è possibile riscontrare - in contrapposizione tra loro.

\footnotetext{
5 Al gruppo di lavoro hanno preso parte: Fabio Bartolazzi, Roberta Bocconcelli, Silvio Cardinali, Elena Cedrola, Marco Cioppi, Giancarlo Ferrero, Giacomo Gisti, Gian Luca Gregori, Fabio Musso, Alessandro Pagano, Tonino Pencarelli, Andrea Perna, Elisabetta Savelli, Valerio Temperini, Annalisa Tunisini.

${ }^{6}$ Le riviste esaminate sono: Economia \& Management, European Journal of Marketing, Journal of Business Strategy, Journal of Business to Business Marketing, Journal of Economics \& Management Strategy, Journal of Marketing, Journal of Marketing Management, Journal of the Academy of Marketing Science, Industrial Marketing Management, Micro \& Macro Marketing, Sinergie.

7 Tale tematica, di particolare interesse in una prospettiva manageriale, è stata solo recentemente approfondita dalla letteratura scientifica (Dewsnap, Jobber 2000; Athens, 2001; Rouziès et al. 2005; Troilo et al. 2009).
} 
La ricerca condotta è di tipo qualitativo e si è basata su casi aziendali di medie imprese. Le indicazioni che ne scaturiscono sono, dunque, circoscritte a questo comparto. La decisione di focalizzare l'indagine su questa tipologia di imprese deriva dall'importanza che esse hanno assunto nell'economia nazionale e dalla complessità delle sfide che devono affrontare nel nuovo contesto economico, considerati da un lato le posizioni di leadership assunte in specifici mercati, dall'altro i limiti strutturali e di competenze manageriali che in molti casi le caratterizzano.

Le medie imprese sono state oggetto di attenzione da parte di ricercatori e di studiosi di varie discipline solo in tempi relativamente recenti, poiché in passato sono state generalmente aggregate alle piccole imprese. E' stato invece correttamente evidenziato in differenti lavori che le medie imprese possono essere efficacemente analizzate come autonomo "oggetto di studio", ricorrendo a specifici approcci metodologici (Varaldo et al., 2010).

Le medie imprese italiane hanno realizzato negli ultimi anni performance mediamente migliori rispetto alle piccole ed alle grandi imprese, evidenziando consistenti percentuali di crescita del fatturato e della redditività (Mediobanca Uniocamere, 2010 e vari anni); si possono individuare tra loro "campioni nascosti" (Simon e Zatta, 2007), che hanno ottenuto risultati eccellenti nei mercati internazionali. A tal proposito, alcuni autori hanno evidenziato la rilevanza di questo "fenomeno", identificando una sorta di "quarto capitalismo"; ciò per sottolineare una diversità sostanziale rispetto all'imprenditorialità fordista e post fordista (Varaldo, 2009; Coltorti, 2010).

E' stato osservato che le medie imprese non hanno il loro punto di forza nella dimensione in quanto tale, ma nell'adeguata combinazione di vari fattori che, in una diversa struttura dimensionale, non troverebbero probabilmente "terreno fertile" per consentire le stesse strategie di crescita (Varaldo et al., 2010).

Pur non esistendo un unico "modello" di media impresa, si possono evidenziare alcuni tratti caratterizzanti e convergenti, relativamente ai processi che creano valore nella prospettiva del cliente. Le attività più rilevanti non sembrano essere quelle strettamente produttive (in vari casi esternalizzate e gestite con un approccio integrato della supply chain). Gli investimenti "strategici" si concentrano piuttosto sui fattori immateriali (conoscenze, brevetti, marchi, pubblicità, progettazione, competenze), che servono ad alimentare l'innovazione ed a favorire il processo di internazionalizzazione; particolare rilevanza assumono, inoltre, il forte legame con i clienti e la capacità di focalizzarsi sulle esigenze di questi, proponendo soluzioni innovative ed incrementando la difficoltà di sostituzione ${ }^{8}$. Analizzare le problematiche e le tendenze evolutive del marketing e delle vendite in questa categoria di imprese aiuta, perciò, a comprendere meglioil particolare approccio al mercato delle imprese italiane d offre elementi per valutare le capacità di risposta alle nuove sfide poste dai mercati.

\footnotetext{
${ }^{8}$ Con riferimento ai modelli di gestione del mercato, Varaldo-Dalli-Resciniti-Tunisini (2010, 213), ne individuano due: "marca-comunicazione-punto vendita” e "prodotto-clienti”, articolati in differenti declinazioni.
} 


\section{Nuove sfide per il marketing dai cambiamenti in atto nel contesto economico}

Dalla metà del 2007 le economie mondiali sono state investite da una crisi finanziaria, commerciale, produttiva, politica e sociale, sfociata in un'ampia recessione che ha bruscamente modificato gli equilibri di mercato esistenti, causando il dissesto di numerosi attori economici. Contestualmente si è ridotto il potere d'acquisto dei consumatori e la loro possibilità di far fronte ai debiti contratti.

Il robusto impatto recessivo mondiale si è manifestato nel 2008 con una decisa caduta degli scambi internazionali (fig. 1) che ha rafforzato la flessione della produzione industriale. Con il ridimensionamento dei redditi e la conseguente diminuzione della domanda, la produzione globale si è ulteriormente ridotta nel corso del 2009.

Fig. 1: Andamento del commercio mondiale 2002-2012 (variazioni annuali percentuali).

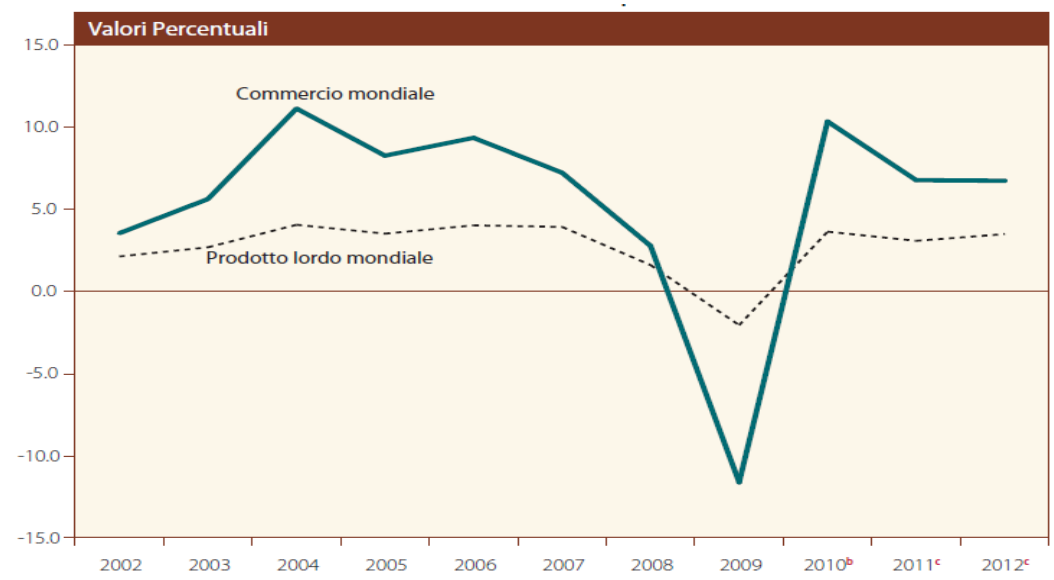

Fonte: United National, Word Economic Situation and Prospect 2011 b: valore stimato - c: proiezione

Gli economisti concordano nel definire il 2009 l'anno in cui la crisi ha raggiunto il punto più basso del ciclo economico (OIC, 2009).

Concomitante alla depressione produttiva è stato un significativo aumento dei licenziamenti e della disoccupazione. Durante un suo intervento al Consiglio Europeo dell'11 Febbraio 2010, Barroso ha dichiarato 23 milioni di disoccupati, 7 milioni in più dei 20 mesi precedenti. Un dato allarmante è quello della disoccupazione giovanile che sarebbe salito al $21 \%^{9}$

A seguito del peggioramento del mercato del lavoro e del diffondersi dell'incertezza, si è assistito ad una riduzione diffusa dei consumi delle famiglie. Un protratto trend

\footnotetext{
${ }^{9}$ http://ec.europa.eu/commission_2010-2014/president/news/statements/pdf/20102010_2_it $(30 / 2 / 2010)$
} 
economico negativo, accompagnato da un generale clima di sfiducia, porta generalmente i consumatori a ridurre gli acquisti, trasformando i "big spenders into penny-pinchers just about everywhere" (Nunes et al, 2009). In base ai dati forniti da A.T. Kearney i settori più colpiti dalla riduzione dei consumi a livello mondiale sarebbero stati quelli dei beni secondari, in particolare delle auto, delle cucine e dei bagni, dei tappeti, delle tende e dei mobili, dei viaggi, del vino e della ristorazione (Walker, 2009).

All'inizio del 2010 la recessione mondiale ha cominciato ad attenuarsi e dai sondaggi d'opinione hanno iniziato ad emergere segnali di minore pessimismo. Ciò ha contribuito a ridurre il grado di ansietà tra i consumatori, nonostante si mantenga ancora a livelli elevati, soprattutto in Spagna e Messico (Roche et al, 2010). Livelli di fiducia maggiori si sono invece registrati in Cina e Brasile.

Dall'osservazione dei principali indicatori congiunturali si evidenzia, inoltre, un ciclo di riprese a due velocità (Vaciago, 2010). Da una parte si collocano le economie in via di sviluppo che, nonostante abbiano pure loro attraversato una fase recessiva, riescono attualmente a conseguire livelli di crescita piuttosto sostenuti, che si prevede raggiungeranno addirittura i 6,5 punti percentuali nel 2011. Dall'altra troviamo i paesi avanzati che mostrano una ripresa dai ritmi decisamente più moderati.

Allo stato attuale, la crisi finanziaria di alcuni paesi, i processi di riorganizzazioni del commercio mondiale, le tensioni politiche che si manifestano in importanti aree geografiche, la crescita dei prezzi delle materie prime continuano a generare una elevata incertezza sul futuro, impedendo di raggiungere una nuova stabilità nei mercati che consenta una più ordinata competizione. Concorrenza e stabilità sono infatti obiettivi complementari sul piano economico, anche se talvolta di complessa coniugazione (Campiglio, 2010).

In questo nuovo contesto economico le imprese necessitano di un rinnovato quadro di governance aziendale e commerciale (Jorek et al, 2009). Il raggiungimento di risultati economici positivi in periodi di profonda trasformazione risulta possibile solo agendo sulle scelte strategiche, sulle funzioni di business (marketing e vendite in primis) e i relativi budget, nonché su un'adeguata gestione del personale. La realtà dei fatti ci presenta invece numerosi casi d'impresa in cui il calo generalizzato delle vendite e della produzione è stato accompagnato da una riduzione degli investimenti in innovazione ed in intangibles, allo scopo di minimizzare costi operativi e di gestione (Ghemawat, 2009). Tale comportamento, per le aziende che hanno deciso di perseguirlo, non ha consentito al management di poter riflettere appieno sugli stimoli provenienti dal mercato e su una reale ridefinizione di struttura e politiche aziendali, per cogliere le nuove opportunità di business.

In particolare, dovrebbe essere proprio in capo alla funzione marketing il compito di mantenere la giusta sintonia con clienti e mercati, favorendo il miglioramento delle performance aziendali. Molte sono le sfide poste a questa funzione dai cambiamenti innescati dalla crisi economica. C'è la necessità di comprendere le nuove connotazioni e la maggiore complessità dei mercati, a seguito delle modifiche intervenute nei criteri di valutazione dei consumatori (Fabris, 2008; Quelch, Jocz, 2009; Oldani, 2009), nelle tendenze dei diversi mercati, nella struttura dell'offerta. Occorre accrescere le capacità di innovazione dei prodotti, facendo leva su strategie 
che valorizzino nuove dimensioni nei processi di creazione del valore (marketing esperienziale, marketing sensoriale, servizi, salvaguardia dell'ambiente, ...) e puntando su riconcettualizzazioni (Normann, 2002; Fortezza, 2008) dei business rivolte alla creazione di nuovi mercati (Kim Chan, Mauborgne, 2005; Valdani, Ancarani, 2009). C'è l'esigenza di stabilire relazioni più durature e cooperative con il trade e con i clienti per coinvolgerli, in logiche di networking (Prahalad, Venkat, 2004), nei processi di creazione del valore. Bisogna saper sfruttare le nuove opportunità offerte dalle ICT. C’è la necessità di realizzare nuove coerenze tra valore offerto e ridotte capacità di spesa dei consumatori. In altri termini il marketing deve sempre più divenire il motore del cambiamento per le imprese, la funzione che più contribuisce a cogliere ed a sfruttare le nuove opportunità che si prospettano.

Un ruolo chiave dovrebbe assumere anche la funzione vendite, per la creazione di relazioni fornitore/cliente di lungo termine. Come primo elemento di contatto col cliente, il personale di vendita ha notevole influenza sulla percezione circa l'affidabilità ed il valore del prodotto e della marca. Da tale influenza dipende non solo l'effettivo collocamento del prodotto, ma anche l'inizio e il consolidamento di una relazione duratura.

Risulta anche sempre più chiaro che uno dei fattori critici per una strategia di vendita di successo è la stretta collaborazione tra funzione marketing e funzione vendite, soprattutto in un clima di crescente competizione come quello attuale (Piercy, 2006). I conflitti che spesso si creano tra le due aree sono da lungo tempo oggetto di interesse e di studio (Dawes e Graham, 2005; Kotler et al, 2006). La loro esistenza e consistenza non stupiscono, considerando la comprensibile distanza concettuale e/o culturale tra coloro che si devono occupare della formulazione e pianificazione di decisioni strategiche orientate al futuro e coloro che operano nella realtà quotidiana, con lo scopo principale di concludere quanti più contratti di vendita possibili. Questa ostilità, o quantomeno scarso coordinamento e collaborazione, ostacola il trasferimento delle conoscenze che i venditori hanno sulla clientela, non contribuendo alla definizione di politiche atte a servire meglio i clienti oggi e a realizzare prodotti migliori in futuro. Risulta quindi necessario dedicare la dovuta attenzione al raggiungimento dell'integrazione delle componenti della sales governance, attraverso l'adozione di una visione olistica del sistema e rafforzando i legami tra i vari comparti d'azienda.

A tal proposito Kotler, Rackham e Krishnaswamy (2006) individuano i requisiti necessari a garantire un efficiente allineamento tra marketing e vendite, ovvero:

- un'adeguata comunicazione tra i team realizzabile attraverso incontri frequenti tra marketing e vendite;

- lo sviluppo di processi precisi, in modo da definire ruoli e responsabilità delle diverse aree e le attività che richiedono la verifica o il coinvolgimento dell'altro gruppo;

- la creazione di opportunità di collaborazione tra i due team, in modo da incrementare la loro capacità di convivenza ed interazione;

- un buon feedback dei venditori con gli addetti al marketing. 
Questi obiettivi rientrano senza dubbio, insieme alla definizione di strutture organizzative più flessibili, tra i requisiti primari per destreggiarsi in contesti incerti e in rapido cambiamento.

\section{Metodologia di indagine e caratteristiche delle imprese intervistate}

L'indagine è stata svolta con interviste semistrutturate effettuate presso quattordici medie imprese di successo delle Marche (tab. 1).

Tab. 1 - Le imprese intervistate

\begin{tabular}{ll}
\hline Nome & Settore \\
\hline Aeffe Spa & abbigliamento \\
Benelli Armi & meccanico \\
Biesse Spa & meccanico \\
Box Marche Spa & cartotecnica \\
FAAM Spa & meccanico \\
FIAM Spa & arredamento \\
Eden Viaggi Srl & turismo \\
Scavolini Spa & arredamento \\
Tessitura Fabbri & tessile/attrezzature balneari \\
Clementoni Spa & Plastica/giocattoli \\
Fileni & alimentare \\
Gruppo Garofoli & arredamento \\
Giano Srl & calzaturiero \\
Loccioni Spa & meccanico-robotica-servizi \\
\hline
\end{tabular}

Il fatto che tutte le imprese intervistate siano localizzate in un'unica regione costituisce certamente un limite dell'indagine. Va tuttavia considerato che la regione Marche può essere considerata rappresentativa di una vasta area del paese (centro Italia, dorsale adriatica) che si è caratterizzata per un forte sviluppo delle piccole medie imprese. Va poi ricordato che la regione Marche, in quanto fortemente industrializzata, ha subito più di altre l'impatto della crisi e, quindi, anche da questo punto di vista può risultare di particolare interesse analizzare come le imprese hanno reagito alla negativa situazione economica.

Abbiamo focalizzato l'attenzione su imprese di successo, sia per avere elementi che ci aiutino ad identificare le best practices di fronte alla crisi, sia per garantire una maggiore generalizzabilità dei risultati, in quanto, essendo molte di queste imprese leader nei rispettivi segmenti, i loro comportamenti condizionano anche le scelte dei più diretti concorrenti.

I soggetti intervistati sono stati, a seconda dei casi, l'imprenditore titolare dell'azienda, il direttore generale, il direttore marketing o più di una di queste figure. I risultati scaturiti dall'indagine rispecchiano, quindi, le opinioni e le percezioni di questi soggetti, che solo per alcuni aspetti che sono state integrate e convalidate sulla 
base di dati oggettivi di carattere quantitativo. Questo costituisce un ulteriore limite dell'indagine svolta.

Le imprese del campione sono tutte imprese familiari, nelle quali l'imprenditore riveste un ruolo centrale nei processi decisionali, anche se nella maggior parte di esse si registra anche la presenza di manager. La media dei fatturati è $€ 133$ milioni, con valori che variano da 9 a 382, il numero medio di addetti è 250 (se si esclude dal calcolo l'impresa più grande che ha 2301 addetti). Come si può vedere nella tabella 1 , i settori rappresentati sono 7 e risultano ampiamente diversificati. Riguardo ai mercati di sbocco, sei imprese hanno per acquirenti altre imprese (BtoB), 8 imprese si rivolgono a mercati di largo consumo $(B t o C)$. L'incidenza dell'export sul fatturato è alta per il 50\% delle imprese intervistate, bassa o nulla per le rimanenti. Solo quattro imprese presentano un grado di diversificazione produttiva significativo.

\section{Le strategie di mercato per fronteggiare la crisi e cogliere le nuove opportunità che si prospettano}

Con riferimento alla crisi economica del biennio 2008-2009, gli interrogativi approfonditi con l'indagine sono stati: in che misura queste imprese "eccellenti" sono state colpite dalla crisi, quali modifiche la crisi ha determinato nei loro mercati di riferimento (congiunturali o strutturali?), quali sono state le risposte di queste imprese alla crisi (tattiche o strategiche? Quali le linee di azione adottate? Allo sviluppo di quali capacità viene dedicata la maggior attenzione?).

Tab.2 - Percentuale delle imprese in base alla dinamica del fatturato nel periodo 2007-2009

\begin{tabular}{lll}
\hline Dinamica fatturato & $2007 / 2008$ & $2008 / 2009$ \\
\hline Calo $>10 \%$ & $7 \%$ & $36 \%$ \\
Calo 5-10\% & $43 \%$ & $14 \%$ \\
Sostanziale stabilità & $36 \%$ & $36 \%$ \\
Crescita sensibile & $14 \%$ & $14 \%$ \\
\hline
\end{tabular}

La recessione economica ha avuto rilevanti riflessi anche su queste imprese, ma meno pesanti che sul complesso del sistema industriale. La metà delle imprese intervistate ha avuto un calo del fatturato sia nel 2008, sia in misura più grave nel 2009 (tab. 2); in circa un terzo delle imprese il fatturato è rimasto stabile; solo due imprese (14\%) hanno registrato un incremento sensibile del fatturato. Per il 2010 la maggior parte delle imprese segnala una decisa ripresa. Considerato il calo della domanda che si è registrato nei mercati di riferimento, si può ipotizzare che la crisi abbia avuto su queste imprese effetti più circoscritti grazie al loro posizionamento in nicchie di mercato, alle solide relazioni instaurate con gli acquirenti ed il trade, alla qualità differenziale dei prodotti ed all'immagine di marca. Questa constatazione ci induce a ritenere che la crisi economica non abbia vanificato la capacità competitiva 
delle medie imprese italiane, anche se lo scenario è articolato e situazioni di forte difficoltà si riscontrano soprattutto nelle imprese meno solide finanziariamente.

Insieme all'impatto quantitativo della crisi, abbiamo cercato di comprenderne l'impatto qualitativo, ovvero di capire quali mutamenti ha determinato nella struttura della domanda, nei criteri di valutazione delle acquirenti, nei comportamenti dei concorrenti. Due sono le indicazioni emerse con riferimento a questi aspetti: 1 . Le imprese non hanno ancora maturato chiari convincimenti sulla natura congiunturale o strutturale delle modifiche che si stanno manifestando nella struttura dei mercati; 2 . le modifiche in atto per alcuni aspetti presentano elementi di omogeneità riscontrabili in tutti mercati, per altri aspetti mostrano differenze significative a seconda del settore.

Numerose sono le imprese che hanno rilevato dinamiche differenziate della domanda a seconda dei segmenti. In generale l'export tira più della domanda interna, anche se si riscontrano differenze sensibili tra le diverse aree geografiche. Molte imprese, tuttavia, hanno sottolineato l'impossibilità di sfruttare le nuove correnti di domanda generate dai paesi del BRIC (o comunque rilevanti difficoltà). Per quanto riguarda la domanda interna, i processi evolutivi risultano diversificati a seconda dei settori. In alcuni mercati (Aeffe, Fiam) si assiste ad una polarizzazione della domanda, che presenta dinamiche di crescita maggiore nei segmenti di fascia alta e in quelli di fascia bassa; in altri casi sono i prodotti high tech (Clementoni, Benelli) ed i segmenti di fascia alta (Scavolini, Fileni) a subire le maggiori contrazioni. Quasi tutte le imprese rilevano una maggiore considerazione per il prezzo, una tendenziale minore fedeltà alla marca, una crescente attenzione per il value for money. Queste tendenze, peraltro, si erano manifestate già negli anni precedenti alla crisi che, quindi, avrebbe accelerato processi evolutivi in atto più che generato cambiamenti inediti. Aumenta la pressione competitiva, anche da parte delle imprese dei paesi emergenti, ma si verificano anche casi (Eden Viaggi, Fileni), in cui il fallimento di importanti concorrenti ha consentito di incrementare le quote di mercato.

A fronte di questi mutamenti nei mercati e dell'incertezza sui loro effetti futuri, la maggior parte delle imprese intervistate ha deciso di adottare risposte non di tipo contingente e tattico, incentrate su tagli di prezzo e forti pressioni sul trade, bensì su strategie rivolte a rafforzare la propria capacità competitiva nel medio termine, dando continuità ai processi evolutivi da tempo avviati, evitando svolte radicali, ponendo grande attenzione a cogliere e sfruttare le nuove opportunità generate dai cambiamenti intervenuti nello scenario economico.

Le linee d'azione adottate sono innanzi tutto dirette ad accrescere l'efficienza e la flessibilità produttiva, intervenendo sulla semplificazione burocratica, sui sistemi di controllo, sulla razionalizzazione della gamma produttiva, sulla logistica, sulla supply chain, sull'introduzione delle ICT, nella consapevolezza che il contenimento dei costi è ormai un imperativo ineludibile. Tra le priorità perseguite con questi interventi c'è anche l'accorciamento dei tempi di risposta ai mutamenti ambientali ed alle richieste dei clienti, considerato ormai fattore critico di successo.

Una grande attenzione è poi dedicata alla ricerca di nuovi duraturi sbocchi di mercato, perseguita, a seconda dei casi, mediante lo sviluppo in nuove aree geografiche (Loccioni, Biesse), l'individuazione di nuove nicchie da sfruttare (Box Marche, Fabbri), l'incremento delle vendite ai clienti tradizionali ed il 
consolidamento delle relazioni intrattenute con essi, una focalizzazione sui segmenti di fascia alta (Fiam, Aeffe), un rafforzamento nei segmenti di mercato più attenti al prezzo (Biesse, Fileni). Vengono dunque perseguite, in alcuni casi congiuntamente, strategie di diversificazione e strategie rivolte ad accrescere la penetrazione nei mercati tradizionali.

Notevole è anche l'impegno dedicato alle strategie di posizionamento. Si punta non a modificare l'immagine di marca ma a rafforzarla e qualificarla mediante le politiche di comunicazione, mediante relazioni più intense con i clienti, mediante la valorizzazione delle linee di prodotto di fascia alta. La qualità del prodotto rimane il principale elemento su cui si fonda il posizionamento di queste imprese, ma si cerca di accrescerne il valore percepito mediante l'offerta di maggiori servizi, una crescente personalizzazione delle prestazioni, un vigile allineamento dei prezzi al valore offerto, una nuova attenzione per gli effetti ambientali delle proprie produzioni.

\section{Il ruolo della funzione di marketing ed i compiti ad essa assegnati}

L'individuazione di questi campi di azione come i più strategicamente rilevanti, mette in luce una nuova, considerevole attenzione verso il marketing e verso le competenze e le professionalità che quest'area funzionale esprime, anche se permane una forte gap tra le esigenze che vengono esplicitate e le risorse che vengono dedicate.

A questo proposito varie solo le considerazioni che emergono dalla ricerca.

Se si analizzano i processi evolutivi in atto si constata un crescente rilievo attribuito al ruolo del marketing, testimoniato: dalla crescita, o comunque dalla stabilità in un quadro di decremento dell'occupazione, degli addetti alla funzione; dall'incremento degli investimenti in marketing in un numero rilevante di imprese (46\%) a fronte di tagli delle risorse dedicate ad altre attività (tab. 3); dall'ampliamento dei campi di intervento della funzione e dell'influenza che essa esercita nei processi decisionali, come staff del vertice aziendale. Se si guarda ai processi di marketing e non solo ai compiti assegnati all'unità organizzativa così denominata, si deve anche rilevare una nuova enfasi attribuita a compiti di marketing (analisi dei clienti e dei concorrenti) assegnati alla funzione vendite.

Tab. 3 - Percentuale delle imprese in base alla dinamica del budget per marketing e comunicazione nel triennio 2007-2010

\begin{tabular}{ll}
\hline Variazione budget & \% imprese \\
\hline Riduzione & $8 \%$ \\
Stabilità & $46 \%$ \\
Lieve aumento & $23 \%$ \\
Forte aumento & $23 \%$ \\
\hline
\end{tabular}


Se però si passa dall'esame delle dinamiche alla fotografia dell'esistente, si osserva che l'unità organizzativa di marketing, pur presente in 12 delle 14 imprese intervistate, ha una consistenza numerica limitata, svolge prevalentemente compiti di supporto (informativi) e di tipo operativo (comunicazione), quasi mai gestisce le leve del prodotto e del prezzo. Le decisioni di marketing di maggior rilievo strategico vengono assunte direttamente dal vertice aziendale, spesso in base alle esperienze passate, a criteri basati sul proprio intuito, ad informazioni acquisite direttamente ed in modo non sistematico.

Passando ad analizzare più in dettaglio come stanno cambiando le finalità ed i compiti assegnati alla funzione di marketing, si deve innanzi tutto sottolineare l'importanza che la maggior parte delle imprese intervistate attribuisce all'obiettivo di accrescere le proprie capacità di marketing intelligence, sia in taluni casi introducendo posizioni organizzative con questo compito specifico, sia, in modo più generalizzato, ridefinendo i compiti degli addetti alle vendite con una forte sottolineatura del ruolo che devono svolgere per la raccolta delle informazioni su i clienti ed i concorrenti. Anche in questo caso tuttavia si constata un gap tra gli obbiettivi enunciati e le tendenze evolutive, da un lato, e la situazione esistente, dall'altro. Le ricerche di mercato formalizzate sono rare, solo alcune imprese dispongono di supporti informatici per un efficace sistema informativo di marketing (es. CRM), pochi sono gli esempi di analisi di customer satisfaction.

Con riferimento alla politica del prodotto, anche se la qualità del prodotto continua ad essere, come sì è detto, uno dei fattori chiave su cui si fonda la competitività di queste imprese, solo in rari casi il marketing ha competenze specifiche in questo campo. Tuttavia, se si considerano le tendenze in atto, si rileva che molte imprese sono sempre più orientate a basare $\mathrm{i}$ processi di innovazione del prodotto più che sull'innovazione tecnologica, sempre importante ma solo in pochi casi fattore critico per acquisire vantaggi concorrenti, sulla comprensione e la soddisfazione di specifiche esigenze dei clienti e del trade al fine di accrescere il valore fornito e percepito, ampliando i servizi, migliorando l'immagine, accorciando i tempi di consegna, personalizzando le prestazioni offerte. Crescente attenzione è inoltre dedicata alla razionalizzazione della gamma per un utilizzo più efficace delle risorse. Si aprono quindi spazi significativi per l'intervento del marketing nella politica del prodotto, che in alcuni casi trovano già concreta realizzazione.

Le politiche di comunicazione rappresentano, nella maggior parte delle imprese intervistate, la principale area di attività dell'ufficio marketing. Ad esse viene attribuita notevole importanza per rafforzare la notorietà e l'immagine del brand. Per questa ragione la maggior parte delle imprese intervistate non ha ridotto gli investimenti in questo campo nonostante la crisi economica, in controtendenza rispetto alle scelte prevalenti a livello nazionale. E' un fatto positivo da sottolineare, unitamente alla sperimentazione di nuove forme di comunicazione nei rapporti BtoB (eventi, open house, ....). Va però anche rilevato che le potenzialità di Internet sono ancora scarsamente utilizzate e che il ricorso a tecniche di marketing non convenzionale, che per almeno alcune di queste imprese potrebbero rivestire notevole interesse, sia sostanzialmente ignorato. Compiti di crescente rilievo nella gestione delle politiche di comunicazione, soprattutto verso il trade, sono attribuiti alle vendite. 
Il prezzo ha assunto un'influenza fortemente crescente nel determinare le scelte dei consumatori. La reazione delle imprese intervistate a questa nuova situazione può essere considerata razionale e coerente con un orientamento gestionale al marketing. Non sì è proceduto a riduzioni generalizzate di prezzo ma si è cercato un corretto rapporto nel value for money; molta cura è stata dedicata ad evitare distorsioni nella percezione del prezzo (accrescendone la trasparenza) e ad assicurare una sua coerenza con il posizionamento del marchio (es. Scavolini). La formulazione delle politiche di prezzo è in genere competenza del vertice aziendale e degli uffici tecnici, ma un ruolo propositivo ed informativo importante è svolto dalle vendite, mentre il marketing, che in passato aveva scarsa voce in capitolo, sta acquisendo un'influenza maggiormente significativa.

Riguardo alle politiche di distribuzione, le linee d'azione adottate sono principalmente rivolte ad obbiettivi di razionalizzazione, con una più chiara differenziazione del ruolo svolto dai diversi canali utilizzati, di potenziamento selettivo per raggiungere una migliore copertura dei mercati-target, di crescita delle capacità di controllo, riducendo il ricorso ad agenti plurimandatari e adottando forme di distribuzione diretta, di rafforzamento delle relazioni con il trade, instaurando rapporti di fiducia e collaborazione. Ancora molto poco utilizzato è il canale Internet. La gestione delle politiche di distribuzione è attribuita alle vendite, talvolta con il supporto del marketing.

Accanto alle politiche del marketing mix, ora analizzate, vanno considerate le politiche di marketing relazionale, che stanno acquisendo importanza sempre più rilevante. Migliorare la gestione dei clienti (il riferimento è soprattutto al trade) è considerato un obiettivo prioritario da molte imprese (Garofoli, Fileni, Benelli, Loccioni, Eden Viaggi). Ciò implica conoscerne meglio le caratteristiche (anche la solidità finanziaria) e le aspettative, fidelizzarli anche con servizi post-vendita, rafforzare i rapporti di fiducia e collaborazione. Lo sviluppo di queste politiche è soprattutto responsabilità delle vendite, mentre il marketing svolge solo un ruolo marginale di supporto.

\section{Il ruolo delle vendite ed il rapporto con il marketing}

Un particolare approfondimento merita l'analisi del ruolo della forza vendita nelle imprese intervistate e delle tendenze evolutive che si manifestano nell'importanza e nei compiti ad essa assegnati.

Nell'indagine empirica si è verificato che vengono adottati modelli di business e modalità di gestione e di organizzazione delle vendite piuttosto differenti.

La funzione vendite risulta da tempo strutturata in queste aziende, assumendo una connotazione più radicata nella organizzazione rispetto al marketing (anche in relazione all'età dei vari addetti); tale funzione svolge ruoli operativi di importanza critica e con ampia autonomia, in modo particolare nel caso di attività realizzate nei mercati internazionali.

Le imprese analizzate hanno progressivamente assegnato alle vendite il ruolo di gestione anche strategica delle relazioni con i clienti; la rete vendita svolge un ruolo 
rilevante nelle azioni di retention, rivolte prevalentemente al trade, anche mediante un processo di "arricchimento" del product offering (con un maggiore contenuto di servizio).

Inoltre, è stato riscontrato che tale funzione contribuisce a soddisfare una rinnovata esigenza informativa relativa al mercato o al singolo cliente (EvansSchlacter, 1985; Festervand-Grove-Reidenbach, 1988); il personale di vendita interviene poi in vario modo nel definire importanti decisioni aziendali.

In molte imprese, quindi, le vendite svolgono un ruolo sempre più critico in ambiti tipici del marketing, quali ad esempio, la scelta dei mercati obiettivo, la definizione delle politiche di prezzo, l'innovazione del prodotto e la gestione della gamma e l'attuazione delle politiche di comunicazione. A ciò corrisponde una crescente importanza assegnata a tale funzione, che costituisce una componente rilevante del capitale relazionale (e del capitale intellettuale nel complesso). Ne deriva che:

- a tale funzione è assegnato un compito anche strategico;

- al personale di vendita è attribuito il ruolo di "value creator", oltre ai compiti tradizionalmente assegnati (Weitz e Bradford, 1999).

Al tempo stesso, si riscontrano varie criticità nella gestione delle forza di vendita, di seguito analizzate ${ }^{10}$.

Vengono di solito adottati sistemi omogenei di gestione delle relazioni con i venditori; al contrario, dovrebbe assumere notevole rilevanza per l'impresa identificare correttamente le caratteristiche del rapporto con la forza vendita, instaurando una politica di gestione coerente con queste. In proposito, non si riscontra una mappatura formalizzata della relazione con i venditori, anche nella prospettiva di delinearne le traiettorie evolutive.

La forza vendita risulta poco coinvolta nella definizione delle zone di vendita e del budget commerciale; ne derivano obiettivi non sempre condivisi.

In vari casi il sistema informativo è inadeguato e non fornisce dati analitici relativi alla attività del singolo venditore; in tale prospettiva, il ricorso al geomarketing sarebbe utile non soltanto per la valutazione delle potenzialità di mercato, ma anche per il monitoraggio della forza vendita.

L'attività di valutazione dei venditori si fonda essenzialmente sulla variabile fatturato; i sistemi di valutazione e di incentivazione della forza di vendita dovrebbero essere invece impostati tenendo conto:

a) dei risultati: considerando però non soltanto il fatturato, o altre variabili "tradizionali" come la quota di mercato ed il numero di visite effettuate, ma anche altri indicatori come, ad esempio, i margini di redditività, il livello di insolvenza dei clienti, gli oneri finanziari figurativi;

b) del comportamento: valutando e misurando gli atteggiamenti, le azioni e le attività che incidono sullo sviluppo dell’impresa e sulla capacità competitiva.

${ }^{10} \mathrm{E}$ ' piuttosto limitato il contributo di studiosi italiani sui vari aspetti del sales management, a differenza di quanto è possibile rilevare a livello internazionale. 
Il ricorso a strumenti di ARM e di Sales Force Automation (SFA) può contribuire ad uno sviluppo delle conoscenze di mercato e ad una maggiore potenzialità di gestione delle relazioni con i venditori ${ }^{11}$.

In una prospettiva di gestione strategica, risulta sempre più opportuno valutare le performance della forza di vendita utilizzando un approccio multidimensionale, nel quale vengono combinati elementi quantitativi e qualitativi, monetari e non monetari. Risulterà opportuno individuare, anche con riferimento al singolo venditore, KPI di nuova generazione quali output dei sistemi informativi integrati.

Non può in conclusione sfuggire che una modalità di gestione della funzione vendita più sistematica e con l'utilizzo dei nuovi sistemi offerti dalle ICT, favorirebbe una naturale connessione e collaborazione con il marketing; ciò richiederebbe però specifiche competenze manageriali che non sempre i responsabili delle vendite sembrano avere.

\section{Considerazioni conclusive}

I casi aziendali esaminati evidenziano una positiva risposta delle medie imprese di successo ai mutamenti generati dalla crisi. Rispetto a scelte difensive e puramente tattiche prevale un approccio proattivo volto a cogliere le opportunità che il nuovo quadro economico può prospettare, senza però adottare svolte radicali, troppo rischiose in una situazione ancora di elevata incertezza. Si nota piuttosto una continuità ed una accelerazione dei processi evolutivi già da tempo avviati, rivolti ad accrescere la capacità di operare in condizioni di crescente complessità gestionale mediante l'inserimento in azienda di nuovi manager (Biesse, Fiam), a creare una maggiore coerenza tra mercati serviti, prodotti offerti e capacità possedute, concentrando le risorse sulle aree dove si dispone di maggiori punti di forza, a sviluppare un più deciso orientamento al mercato per accrescere le capacità di innovazione e per cogliere nuove possibilità di sviluppo.

L’importanza di attuare un riposizionamento oltre la crisi e di delineare nuove strategie di sviluppo hanno indotto le medie imprese analizzate ad attribuire un nuovo ruolo al marketing, che risulta essere maggiormente valorizzato. Si nota una crescente attenzione verso la raccolta e l'analisi delle informazioni sui mercati ed i clienti, una focalizzazione sugli attributi ed i processi che contribuiscono in modo più rilevante a creare valore per il consumatore ed a farglielo correttamente percepire, un rinnovato impegno verso lo sviluppo di relazioni solide e cooperative verso il trade ed i migliori clienti.

Si afferma un più deciso orientamento strategico nei processi decisionali delle funzioni di marketing e di vendita, migliora la qualificazione professionale degli addetti a queste funzioni, cresce la collaborazione e l'integrazione tra di esse. Molte

11 Il modello ARM prende spunto dai contributi teorici sulla Sales Force Automation e sul CRM; si veda in proposito Gregori et al. (2009). 
sono le decisioni assunte congiuntamente, il marketing supporta le vendite nei compiti operativi, le vendite supportano il marketing nelle attività di analisi.

Il ruolo più importante nella valutazione dei mercati e nella definizione delle strategie commerciali continua comunque ad essere svolto, nella quasi totalità delle imprese esaminate, dal vertice aziendale (imprenditore o amministratore delegato), che integra le indicazioni che gli provengono dal marketing e dalle vendite con altri elementi tratti dalla sua esperienza, dà suoi canali informativi, da sue intuizioni.

Va, quindi, osservato che nelle imprese esaminate la funzione di marketing si configura come una funzione diffusa, che coinvolge:

- il vertice aziendale;

- la direzione marketing;

- la direzione vendite.

Solo un esame congiunto delle attività svolte da queste tre unità organizzative consente una corretta valutazione dei processi di marketing gestiti dall'impresa. La ricerca evidenzia un tendenziale passaggio da un approccio ai mercati di tipo intuitivo, poco strutturato, basato in larga parte sulle esperienze passate, gestito prevalentemente dal vertice aziendale, con compiti assegnati alle vendite ed al marketing quasi esclusivamente di tipo esecutivo-operativo nell'ambito di ruoli differenziati e scarsamente interagenti, ad un approccio più strutturato ed organico, che si fonda su un maggiore coinvolgimento del marketing e delle vendite nei processi decisionali anche di tipo strategico, su rapporti di intensa collaborazione tra queste funzioni e su un rafforzamento delle risorse umane e finanziarie ad esse assegnate.

Va tuttavia precisato che si tratta di tendenze evolutive che richiedono, per giungere a compimento, ancora molta strada da percorrere. Al momento si riscontra una significativa distanza tra le finalità che si vorrebbero perseguire e le risorse ed i poteri decisionali attribuiti a queste funzioni. I confini operativi del marketing rimangono troppo ristretti, sono ancora poco sfruttate varie possibilità di innovazione connesse ad internet ed a Web 2.0, rari sono gli investimenti diretti a garantire supporti informatici adeguati per una gestione delle informazioni e dei clienti più razionale e strutturata (es. CRM), scarso è l'utilizzo di forme di comunicazione non convenzionale. Analoghe considerazioni possono essere svolte con riferimento alle vendite, per le quali all'ampliamento ed alla qualificazione del ruolo attribuito non corrispondono ancora coerenti sistemi di valutazione ed incentivazione, sistemi operativi di tipo informatico adeguati, una appropriata qualificazione delle capacità manageriali del personale.

In conclusione, si rileva un percorso evolutivo in atto, che ha subito una decisa accelerazione con la crisi economica, verso un orientamento al mercato da parte di queste imprese, supportato da una valorizzazione, un rafforzamento ed una ridefinizione dei ruoli delle funzioni di confine con i mercati di sbocco (marketing e vendite). Questo percorso è tuttavia ancora fortemente ostacolato da vincoli culturali e di risorse, anche se cresce la consapevolezza nei vertici aziendali che tali ostacoli 
dovranno essere superati per affrontare efficacemente le nuove sfide che si prospettano all'orizzonte.

\section{Riferimenti bibliografici}

Athens D. (2001), Integration Between Marketing and Sales, Chicago: American Marketing Association.

Campiglio L. (2010). Quale concorrenza dopo la crisi, in AA.VV. Dopo la crisi: conseguenze economiche, finanziarie e sociali, Milano: Giuffrè.

Carpenter P. (1992), Bridging the Gap between Marketing and Sales, Marketing and Sales Management, 144 (March), 29-31.

Coltorti F., 2010, “Crisi, quarto capitalismo e competitività delle medie imprese”, Prolusione MBA CUOA, Luglio, Fondazione Cuoa,

Dawes P. L. and Graham R. M (2005). Antecedents of Conflict in Marketing's Cross-Functional Relationship with Sales. European Journal of Marketing: 14, 11-12, 1327-1344.

Dawes P.L., Massey G.R. (2005), Antecedents of Conflict in Marketing’s CrossFunctional Relationship with Sales, European Journal of Marketing, 14 (11-12), 1327-1344.

Dewsnap B., Jobber D. (2000), The Sales-Marketing Interface in Consumer Packaged-Goods Companies: A Conceptual Framework, Journal of Personal Selling and Sales Management, 20, 2(Spring), 109-119.

Evans K.R., Schlacter J.L. (1985), The role of Sales Managers and Salespeople in Marketing Information System, Journal of Personal Selling and Sales Management, 5, 2 (November), 49-55.

Fabris GP. (2008), Societing, Milano: Egea

Ferrero G., Fortezza F. (2007), Importanza ed elementi di criticità del marketing strategico nelle PMI, Piccola Impresa - Small Business, n. 2

Festervand T.A., Grove S.J., Reidenbach R.E. (1988), The sales Force as a Marketing Intelligence System, Journal of Business and Industrial Marketing, 3 (1), 53-59.

Fortezza F. (2008), Processi strategici e di marketing nel settore della nautica da diporto, Milano: F. Angeli

Ghemawat P. (2009). The Risk of Not Investing in a Recession. MIT Sloan Management Review: 50, 3, 31-38.

Gregori G.L., Cardinali S., Temperini V., Perna A. (2009), "Forza di vendita indiretta e capitale relazionale", paper presentato al $32^{\circ}$ Convegno AIDEA (Convegno Annuale dell'Accademia Italiana di Economia Aziendale), Ancona, 24-25 settembre.

Guenzi P., Troilo G. (2007), The Joint Contribution of Marketing and Sales to the Creation of Superior Customer Value, Journal of Business Research, 60(2), 98-107.

Homburg C., Jensen O. (2007), The Thought Worlds of Marketing and Sales: Which Differences Make a Difference?, Journal of Marketing, 71(July), 124-142. 
Jorek N., Battat M. and Rajendran V. (2009). Global Champion 2009. A.T. Kearney, White Paper, testo disponibile al sito http://www.atkearney.com/index.php/Publications/the-at-kearney-global-champions2009.html (ultimo accesso/24/02/2011).

Kim W. Chan, Mauborgne R. (2005), Blu Ocean Strategy. How to CreateUncontested Market Space and Make the Competition Irrelevant, Boston: Harvard Business School Press

Kotler P., Rackham N. and Krishnaswamy S. (2006). Basta con le guerre tra Vendite e Marketing. Harvard Business Review Italia: 3, luglio/agosto, 25-45.

LeMeuneir-FitzHugh K., Piercy N. (2006), Integrating Marketing Intelligence Sources: Reconsidering the Role of the Sales force, International Journal of Marketing Research, 48 (6), 699-716.

Marchini I. (2000), Il governo della piccola impresa. La gestione delle funzioni, Vol. III, Genova: Aspi/Ins-Edit.

Mattiacci A., Ceccotti F. (2005), Lo sviluppo del marketing nella Pmi: riflessioni a margine di un'esperienza d'impresa, Micro \& Macro Marketing, n. 1.

McCartan-Quinn D., Carson D. (2003), Issues which Impact upon Marketing in the Small Firm, Small Business Economics, n. 21.

Normann R. (2002), Le condizioni di sviluppo dell'impresa, Milano: Etas Libri

Nunes P. F., Polka C. J. and Thomas L. (2009). How to make the most of the great consumer trade down. Accenture Outlook, June, testo disponibile al sito http://www.accenture.com/us-en/research/institute-high-performance/pages/insightshow-make-most-great-consumer-trade-down.aspx (ultimoaccesso/24/02/2011).

OIC (2009). "Sesric Monthly Reports on the Global Financial Crisis of 20082009”, August/September 2009, testo disponibile al sito http://www.sesric.org/files/article/373.pdf (ultimoaccesso/30/10/2010).

Oldani F., L’impatto della crisi attuale condizionerà i consumi di domani, Mark Up, 2009 3-28.

Piercy N. F. (2006). The Strategic Sales Organization. The Marketing Review: 6,

Prahalad C.K., Venkat Ramaswamy, (2004), Il futuro della competizione. Cocreare valore eccezionale per i clienti, Milano: Il Sole 24 ORE Libri

Quelch J.A., Jocz K.E. (2009), Il marketing al tempo della recessione, Piccola biblioteca del Sole 24 ORE, n. 19

Roche C., Ducasse P., Liao C. and Grevler C. (2010). “A New World Order of Consumption. Customer in a Turbolent Recover, in The Boston Consulting Group”, 2010 BCG Global Report on Consumer Sentiment. June, testo disponibile al sito http://www.bcg.it/expertise_impact/Industries/Tecnologia_Media_e_Telecomunicazio ni/Publication Details.aspx?id=tcm:129-51388\&mid= (ultimo accesso/24/02/2011).

United Nation (2011), World Economic Situation and Prospect 2011. New York, testo disponibile al sito http://www.un.org/en/development/desa/policy/wesp (ultimoaccesso/24/2/2011).

Vaciago G. (2010). "Sintesi”, in Osservatorio Monetario ASSBB: 2., testo disponibile al sito http:www.assbb.it/laboratorio/osservatorio.asp (ultimoaccesso/30/10/2010). 
Valdani E., Ancarani F. (2009), Marketing strategico, Milano: Egea

Varaldo R., Dalli D., Resciniti R. (2006), "Marketing-non-marketing all'italiana: virtù, limiti e prospettive”, Paper presentato al Congresso Internazionale su «Le Tendenze del Marketing», 20-21 Gennaio.

Varaldo R., Dalli D., Resciniti R., Tunisini A. (2009), Un tesoro emergente: Le medie imprese italiane dell'era globale, Milano: F. Angeli

Walker M. (2009). “2009 Outlook. Difficult times will continue this year”. A.T. Kearney, White Paper, testo disponibile al sito http://www.atkearney. com/index.php/Publications/2009-utlook.html?q=2009+outlook (ultimoaccesso/24 /02/ 2011). 